\title{
PROTON STRUCTURE AND PDFs FROM HERA*
}

\author{
PAVEl Belov \\ on behalf of the H1 and ZEUS collaborations \\ DESY, Notkestrasse 85, 22607 Hamburg, Germany
}

(Received January 25, 2013)

\begin{abstract}
During the past years, measurements of the proton structure at the HERA $e p$ collider have steadily improved in precision. The most recent results on the proton structure and parton densities are presented. The low $Q^{2}$ measurements of the longitudinal structure function $F_{\mathrm{L}}$ and the comparison to phenomenological low $x$ models are described.
\end{abstract}

DOI:10.5506/APhysPolBSupp.6.655

PACS numbers: $13.60 . \mathrm{Hb}$

\section{Introduction}

Measurements of inclusive differential cross sections in Deep Inelastic Scattering (DIS) are crucial to determining the proton structure. The structure functions provide detailed information on the Parton Density Functions of the proton (PDFs) which play an important role for predictions at the proton-proton collider LHC. A dominant part of the data used to determine the PDFs comes from the electron(positron)-proton HERA collider, which was operating at DESY from 1993 until 2007. The HERA collider opened up a wide kinematic range for precision measurements of the proton structure due to the high centre-of-mass energies of interactions, $\sqrt{s}=225-318 \mathrm{GeV}$, and large integrated luminosities. The kinematic range accessible by the HERA experiments, H1 and ZEUS, is [1] $0.01<Q^{2}<3 \times 10^{4} \mathrm{GeV}^{2}$ for the absolute four momentum transfer squared and $10^{-6}<x<0.65$ for the Bjorken- $x$ variable, which is the fraction of the proton momentum carried by the struck parton. The precision HERA data constrain the PDFs at low to medium $x$, whereas the fixed target and hadro-production experiments contribute mainly at high $x$.

* Presented at the International Symposium on Multiparticle Dynamics, Kielce, Poland, September 17-21, 2012. 


\section{Parton density functions}

The DIS process in which the electron is scattered off the proton via exchange of a $\gamma$ or $Z^{0}$-boson (neutral current process) or via exchange of a $W^{ \pm}$-boson (charge current process) is investigated in the following. The kinematics of the presented $e p$ DIS process is expressed by three invariant variables: Bjorken- $x$, boson virtuality $Q^{2}$, and inelasticity $y$. The neutral current $(\mathrm{NC})$ double differential cross section is decomposed into the proton structure functions $F_{2}, F_{\mathrm{L}}$ and $x F_{3}$

$$
\frac{d^{2} \sigma_{\mathrm{NC}}^{ \pm}}{d x d Q^{2}}=\frac{2 \pi \alpha^{2}}{x Q^{4}}\left[Y_{+} F_{2} \mp Y_{-} x F_{3}-y^{2} F_{\mathrm{L}}\right],
$$

where the factor $Y_{ \pm}=1 \pm(1-y)^{2}$ stands for helicity dependence. The $F_{2}$ term is dominant. At leading order (LO), it is given by the chargesquared weighted sum of the quark and anti-quark densities (sea quarks): $F_{2}=x \sum_{q} e_{q}^{2}(q+\bar{q})$. The $x F_{3}$ term is related to the difference of the quark and anti-quark densities (valence quarks): $x F_{3}=x \sum_{q} 2 e_{q} a_{q}(q-\bar{q})$, and becomes important at high $Q^{2}$. The longitudinal structure function $F_{\mathrm{L}}$ is zero at $\mathrm{LO}$, but is predicted to be non-zero at higher orders in QCD due to gluon emissions.

Similar to NC scattering, the inclusive charge current (CC) ep cross section can be expressed in terms of PDFs. At LO, the $e^{+} p$ and $e^{-} p$ inclusive $\mathrm{CC}$ cross sections are sensitive to different combinations of quark and antiquark densities.

The PDFs can be determined from the inclusive NC and CC measurements. At HERA, the cross section data measured by the H1 and ZEUS collaborations have been combined using a method [2], which takes into account the correlations of the systematic uncertainties. A QCD fit to the data for $Q^{2}>3.5 \mathrm{GeV}^{2}$ is performed for obtaining the PDFs. The fitted PDFs are $x g, x u_{v}, x d_{v}, x \bar{U}, x \bar{D}$, where $x \bar{U}=x \bar{u}, x \bar{D}=x \bar{d}+x \bar{s}$. These input PDFs are parametrized at the evolution starting scale $Q_{0}^{2}=1.9 \mathrm{GeV}^{2}$ using the following functional form

$$
x f(x)=A x^{B}(1-x)^{C}\left(1+D x+E x^{2}\right),
$$

where the normalization parameters $A_{u_{v}}, A_{d_{v}}, A_{g}$ are constrained by the quark counting and momentum sum rules. The relation $B_{\bar{U}}=B_{\bar{D}}$ guarantees that $x \bar{u} \rightarrow x \bar{d}$ as $x \rightarrow 0$. The PDFs are evolved using DGLAP evolution equations [3] at NLO [4] and NNLO [5] in the $\overline{\mathrm{MS}}$ scheme. The renormalization and factorization scales are chosen equal to $Q^{2}$, and the strong coupling $\alpha_{\mathrm{s}}\left(M_{Z}\right)=0.1176$. A QCD predictions for the structure functions are obtained by convoluting the PDFs with the coefficient functions, which are calculated using the ACOT [6] and RT [7] heavy flavour schemes. 
In the fit, the correlations induced by systematic uncertainties are properly taken into account. Besides the experimental uncertainties, model and parametrization uncertainties are determined by varying model inputs and parameters of (2). The model uncertainties are obtained by a variation of the minimum cut $Q_{\min }^{2}$, the charm and beauty mass parameters, and the strangeness fraction. The parametrization uncertainties are estimated by including additional parameters into the functional form (2) and by varying the starting scale $Q_{0}^{2}$.

\section{HERAPDF}

HERAPDF is a series of the PDF sets released by the H1 and ZEUS collaborations. A preliminary combination of the full HERA datasets of the inclusive $\mathrm{NC}$ and $\mathrm{CC}$ cross sections [8] have been used to determine the HERAPDF1.5 set [9]. The inclusion of more high $Q^{2}$ data as compared to [1] significantly increases the statistics and leads to a more precise description of the PDFs at high $x$. The HERAPDF1.5 set is available at LHAPDF and is currently recommended by the $\mathrm{H} 1$ and ZEUS collaborations. The newest released PDF set is HERAPDF1.7 [10]. It includes all currently available HERA NC and CC inclusive, charm [11], low energy [12, 13] and jet data [14] which have been used in the NLO QCD fit. The extracted PDFs are shown in Fig. 1. The PDFs are strongly constrained and consistent with previous results of HERAPDF1.6 [15], which do not include charm data.

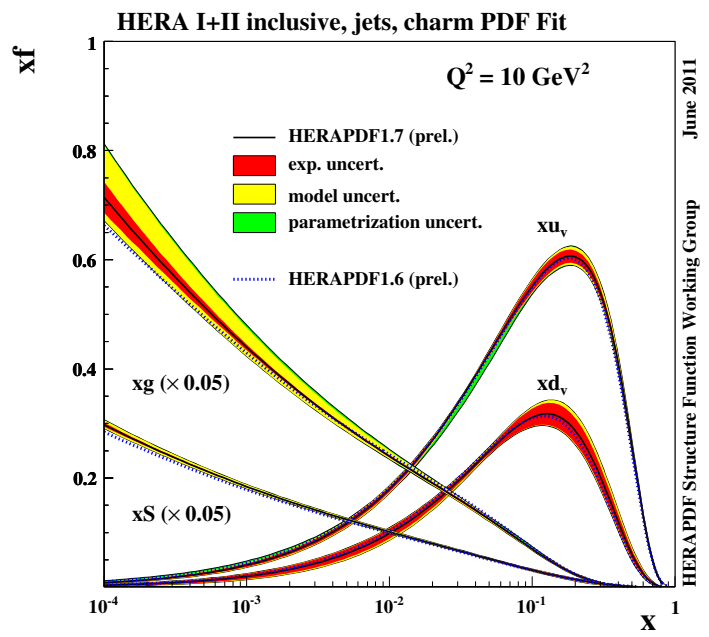

Fig. 1. The PDFs from HERAPDF1.7 for $Q^{2}=10 \mathrm{GeV}^{2}$. The gluon $(x g)$ and sea $(x S)$ densities are scaled down by a factor of 20 . The central values of HERAPDF1.6 are also shown for comparison. 
The combined data have been used for a comparison of the PDFs obtained at different orders of perturbation theory. This comparison is presented in Fig. 2. Both fits are done using the same sufficiently flexible gluon parametrization, such that the fit works with both NLO and NNLO QCD evolutions. The obtained $\chi^{2} / n_{\text {dof }}$ is similar for both fits. At low $x$, the model uncertainty for the NNLO fit is larger. It means that the NNLO fit is more sensitive to the low $Q^{2}$ data, which at HERA correspond to the low $x$ region. This study may indicate that the low $Q^{2}$ and low $x$ data contain contributions beyond the standard perturbation expansion and so need to be considered in more details in the future.
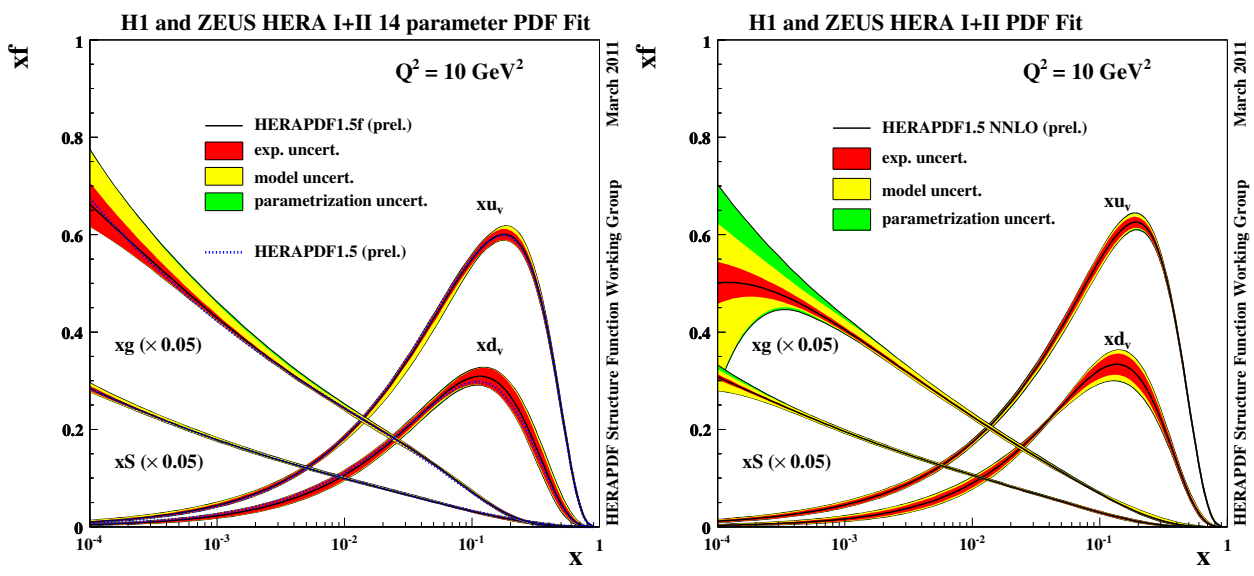

Fig. 2. The PDFs obtained from a QCD fit at different orders of perturbation theory, NLO (left), NNLO (right), are shown for $Q^{2}=10 \mathrm{GeV}^{2}$. The gluon $(x g)$ and sea $(x S)$ densities are scaled down by a factor of 20 . The HERAPDF1.5 preliminary fit which has less parameters for $x g$ is also shown for comparison.

\section{4. $F_{\mathrm{L}}$ measurements}

One of the most interesting low $x$ measurements is the measurement of the longitudinal structure function $F_{\mathrm{L}}$. This measurement uses the data collected during nominal, $E_{p}=920 \mathrm{GeV}$, proton beam energy runs, as well as during dedicated low energy, $E_{p}=460,575 \mathrm{GeV}$, runs. The inclusive $\mathrm{NC}$ cross section measurements have been performed for the same values of $Q^{2}$ and $x$, but different inelasticities $y=Q^{2} /(s x)$. That made it possible to measure $F_{\mathrm{L}}$ for each $\left(x, Q^{2}\right)$ point. Measurements have been carried out by the H1 and ZEUS collaborations [12, 13] and a good agreement between experiments has been observed. The obtained values together with the predictions of various PDF groups are shown in Fig. 3. For $Q^{2}>10 \mathrm{GeV}^{2}$, the measured data confirm the theory predictions, whereas for $Q^{2}<10 \mathrm{GeV}^{2}$, 
different groups predict different low $Q^{2}$ behaviour of $F_{\mathrm{L}}$. Unfortunately, large experimental uncertainties prevent any strong conclusion about the low $Q^{2}$ behaviour of $F_{\mathrm{L}}$.

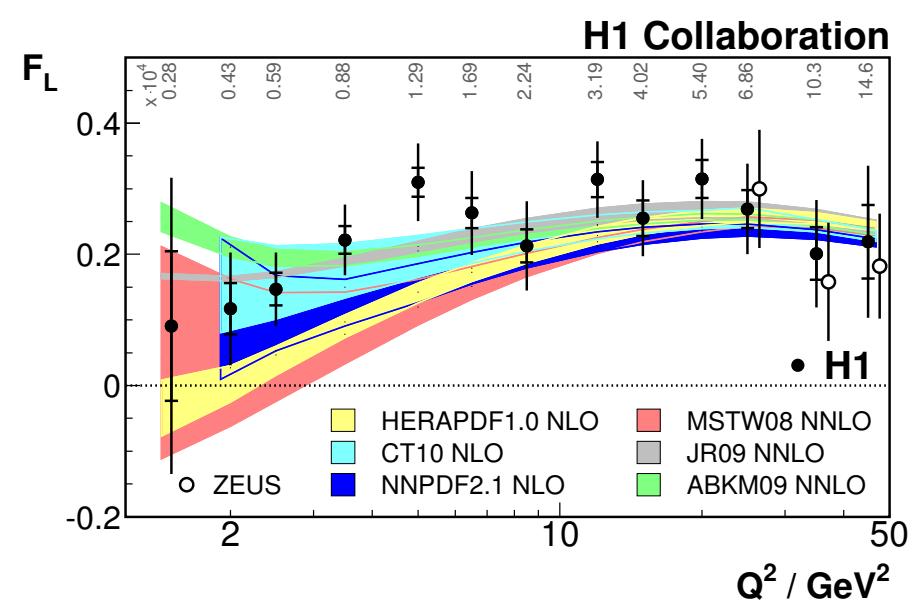

Fig. 3. The longitudinal structure function $F_{\mathrm{L}}$ shown as a function of $Q^{2}$ and average $x$ values of each $Q^{2}$. The inner error bars represent statistical uncertainties, whereas the full error bars show total uncertainties.

\section{Comparison to phenomenological low $x$ models}

At low $x$ and low $Q^{2}$, the structure functions can be described using the colour dipole model formalism [16]. The dipole models assume a factorization of the photon-proton scattering process into a photon splitting and scattering of the obtained $q \bar{q}$ pair off the proton target. Three models have been confronted to HERA data: the original model version (GBW) [17], a model based on the colour glass condensate approach to the high parton density regime (IIM) [18], and a modified GBW model, adding effects of the DGLAP evolution (B-SAT) [19]. The dipole models assume gluon dominance, valence quark contributions are ignored. However, the PDFs suggest that these contributions reach $10 \%$ for $x \sim 0.01$. For the data comparison [12], pure dipole model fits, DGLAP fits, and the dipole model fits with the valence contributions estimated from the DGLAP fit are performed in the kinematic region $x<0.01$ and $Q^{2}>3.5 \mathrm{GeV}^{2}$. The NLO DGLAP fit in the ACOT scheme as well as the IIM dipole model fit show the best description of the data with $\chi^{2} / n_{\text {dof }}=248.3 / 249$ and $\chi^{2} / n_{\text {dof }}=259.4 / 252$, respectively. Other dipole models describe the data less well. Interestingly enough, the addition of valence contributions degrades the fit quality. 


\section{Conclusions}

The recent HERA data strongly constrain the parton density functions. The measurements at lower centre-of-mass energies made it possible to obtain the longitudinal structure function $F_{\mathrm{L}}$. The phenomenological low $x$ models describe the measurements well.

\section{REFERENCES}

[1] H1 and ZEUS collaborations, J. High Energy Phys. 1001, 109 (2010).

[2] A. Glazov, AIP Conf. Proc. 792, 237 (2005).

[3] G. Altarelli, G. Parisi, Nucl. Phys. B126, 298 (1977); Yu.L. Dokshitzer, Sov. Phys. JETP 46, 641 (1977); V.N. Gribov, L.N. Lipatov, Sov. J. Nucl. Phys. 15, 438 (1972); 15, 675 (1972); L.N. Lipatov, Sov. J. Nucl. Phys. 20, 94 (1975).

[4] G. Curci, W. Furmanski, R. Petronzio, Nucl. Phys. B175, 27 (1980); W. Furmanski, R. Petronzio, Phys. Lett. B97, 437 (1980).

[5] S. Moch, J. Vermaseren, A. Vogt, Nucl. Phys. B688, 101 (2004); A. Vogt, S. Moch, J. Vermaseren, Nucl. Phys. B691, 129 (2004).

[6] M. Kramer, F.I. Olness, D.E. Soper, Phys. Rev. D62, 096007 (2000).

[7] R.S. Thorne, R.G. Roberts, Phys. Rev. D57, 6871 (1998); R.S. Thorne, Phys. Rev. D73, 054019 (2006).

[8] H1 and ZEUS collaborations, H1prelim-10-141, ZEUS-prel-10-017.

[9] H1 and ZEUS collaborations, H1prelim-11-042, ZEUS-prel-11-002.

[10] H1 and ZEUS collaborations, H1prelim-11-143, ZEUS-prel-11-010.

[11] H1 and ZEUS collaborations, H1prelim-10-143, ZEUS-prel-10-019.

[12] H1 Collaboration, Eur. Phys. J. C71, 1579 (2011).

[13] ZEUS Collaboration, Phys. Lett. B682, 8 (2009).

[14] H1 Collaboration, Eur. Phys. J. C65, 363 (2010); C67, 1 (2010); ZEUS Collaboration, Phys. Lett. B547, 164 (2002); Nucl. Phys. B765, 1 (2007).

[15] H1 and ZEUS collaborations, H1prelim-11-034, ZEUS-prel-11-001.

[16] N.N. Nikolaev, B.G. Zakharov, Z. Phys. C49, 607 (1991).

[17] K. Golec-Biernat, M. Wüsthoff, Phys. Rev. D59, 014017 (1999).

[18] E. Iancu, K. Itakura, S. Munier, Phys. Lett. B590, 199 (2004).

[19] H. Kowalski, L. Motyka, G. Watt, Phys. Rev. D74, 074016 (2006). 